\title{
The effects of miR-140-5p on the biological characteristics of ovarian cancer cells through the Wnt signaling pathway
}

\author{
Yunyu Wu ${ }^{1, A, B, E, F}$, Jie Li ${ }^{2, B-F}$, Shuying Chen ${ }^{3, B-D}$, Zhiwu Yu ${ }^{3, A-C}$ \\ ${ }^{1}$ Department of Gynecology, Affiliated Cancer Hospital \& Institute of Guangzhou Medical University, China \\ 2 Department of Intensive Care Unit, Guangdong Provincial Geriatrics Institute, Guangdong Provincial People's Hospital, Guangdong Academy of Medical Sciences, Guangzhou, \\ China \\ ${ }^{3}$ Department of Clinical Laboratory, Affiliated Cancer Hospital \& Institute of Guangzhou Medical University, China \\ A - research concept and design; $\mathrm{B}$ - collection and/or assembly of data; $\mathrm{C}$ - data analysis and interpretation; \\ $D$ - writing the article; $E$ - critical revision of the article; $F$ - final approval of the article
}

Address for correspondence

Zhiwu Yu

E-mail:yuzhiwu1yzw7@163.com

\section{Funding sources}

This work was supported by the Medical Health Science and Technology Project of Guangzhou City (grant No. 20161A010087).

\section{Conflict of interest} None declared

Received on January 2,2020

Reviewed on February 13, 2020

Accepted on May 1, 2020

Published online on August 4, 2020

\section{Cite as}

Wu Y, Li J, Chen S, Yu Z. The effects of miR-140-5p on the biological characteristics of ovarian cancer cells through the Wnt signaling pathway. Adv Clin Exp Med. 2020;29(7):777-784. doi:10.17219/acem/121933

\section{DOI}

10.17219/acem/121933

\section{Copyright}

Copyright by Author(s)

This is an article distributed under the terms of the Creative Commons Attribution 3.0 Unported (CC BY 3.0) (https://creativecommons.org/licenses/by/3.0/)

\begin{abstract}
Background. Ovarian cancer is usually not diagnosed until the late stage, and it is resistant to platinum and other standard chemotherapy drugs, resulting in high mortality.

Objectives. To investigate the effects of miR-140-5p on cell proliferation, apoptosis, invasion, and migration capability in the SKOV3 and OVCAR3 ovarian cancer cell lines through Wnt signaling pathway.

Material and methods. Expression levels of miR-140-5p were checked using quantitative real-time polymerase chain reaction (qRT-PCR). The expression of miR-140-5p was upregulated by transfecting cells with a miR-140-5p mimic or a mimic negative control (NC). Cell proliferation was assessed using a CCK8 assay, and cell cycle distribution and apoptosis percentage were detected with flow cytometry. A transwell invasion assay was employed to evaluate cell migration and invasion ability. The target complementary relationship between miR-140-5p and WNT7 was verified using a dual-luciferase reporter assay while $\beta$-catenin in the nuclei was observed using immunofluorescence. The expression of Wnt signaling pathway-related proteins was examined using western blot and gRT-PCR.
\end{abstract}

Results. The relative expression level of miR-140-5p in SKOV3 and OVCAR3 cells was obviously decreased compared with that in the IOSE80 cells $(p<0.05)$. Besides, upregulated miR-140-5p effectively suppressed cell proliferation and increased the apoptosis ratio of SKOV3 and OVCAR3 cells $(p<0.05)$. In addition, the invasion and migration capability of SKOV3 and OVCAR3 cells in miR-140-5p mimic group was largely suppressed compared with the NC group $(p<0.05)$. What is more, the target complementary relationship between miR-140-5p and the WNT1 gene was revealed; upregulated miR-140-5p suppressed the expression of Wnt signaling-related genes, and restrained nuclear transfer of $\beta$-catenin $(p<0.05)$.

Conclusions. The overexpression of miR-140-5p restricted the proliferation, migration and invasion abilities, and accelerated cell apoptosis in ovarian cancer cell lines SKOV3 and OVCAR3 through the Wnt signaling pathway.

Key words: apoptosis, ovarian cancer, cell proliferation, Wnt signaling pathway, miR-140-5p 
Ovarian cancer has the $7^{\text {th }}$ highest incidence and $8^{\text {th }}$ highest fatality rate among malignant gynecological diseases in the world, with a five-year survival rate lower than $45 \% .{ }^{1-4}$ At present, the most common therapy for ovarian cancer is surgical resection of visible lesions, combined with chemotherapy using targeted platinum drugs (such as cisplatin or carboplatin) and paclitaxel. ${ }^{5}$ However, longterm use of these chemotherapy regimens carries the risk of drug resistance. Various advances in surgical technology and chemotherapy have been made in recent years. However, instead of early detection, ovarian cancer is usually not diagnosed until the late stages, and it is resistant to platinum and other standard chemotherapy drugs, thus resulting in high mortality. ${ }^{6,7}$ Therefore, it is urgent to explore new and effective treatments for ovarian cancer.

Gene therapy, especially microRNA-regulated gene therapy, has attracted extensive attention recently years. ${ }^{8}$ MicroRNAs (miRs) are able to regulate cell proliferation, differentiation and apoptosis, as well as the occurrence and development of cancer. ${ }^{9}$ Regulation of miR expression has provided important ideas for the prevention and control of a variety of malignant diseases, including papillary thyroid cancer, prostate cancer, breast cancer, non-small cell lung cancer (NSCLC), and so on. ${ }^{10-13}$ For example, miR140-5p overexpression has effectively inhibited the growth, metastasis, invasion, and epithelial-mesenchymal transformation of hepatocellular carcinoma (HCC) and NSCLC. ${ }^{14,15}$ However, the effects of miR-140-5p on ovarian cancer have not been clearly illustrated. We learned from a biological information website that complementary binding sites exist between miR-140-5p and the WNT1 gene, and we know that WNT1 is an important gene in the Wnt signaling pathway, whose abnormal expression plays a key regulation function in the onset and progress of many cancers. ${ }^{16}$

The aim of our study was to research the expression of miR140-5p and the WNT1 gene in ovarian cancer cells, and the effects of miR-140-5p on the biological characteristics of ovarian cancer cells through the Wnt signaling pathway.

\section{Material and methods}

\section{Cell culture and transfection}

The human epithelial ovarian cancer SKOV3 and OVCAR3 cell lines used here were originally purchased from the ATCC cell bank (Manassas, USA). Human normal ovarian epithelial cells IOSE80 were acquired from the cell bank of the Chinese Academy of Sciences (Shanghai, China). The cells were kept in RPMI 1640 medium with $10 \%$ fetal bovine serum (FBS), $100 \mu \mathrm{g} / \mathrm{mL}$ of streptomycin and $100 \mathrm{U} / \mathrm{mL}$ of penicillin (Gibco BioSciences, Dublin, Ireland) at $37^{\circ}$ in $5 \% \mathrm{CO}_{2}$, and the cells were not sub-cultured until cell density was up to $80 \%$.

SKOV3 and OVCAR3 cells at the logarithmic growth stage were inoculated into six-well plates $\left(5^{*} 10^{5}\right.$ cells $\left./ \mathrm{mL}\right)$ and were then co-transfected with miR-140-5p mimic or mimic negative control (NC) (Gemma Biological Co., Ltd., Shanghai, China) using Lipofectamin 2000 (Invitrogen Corp., Carlsbad, USA) following standard instructions.

\section{Dual-luciferase reporter assay}

Binding sites between miR-140-5p and WNT1 were first predicted on the Bioinformatics \& Research Computing website (www.targetscan.org) and the fragment sequence containing the binding sites was obtained. Synthetic WNT1-WT or WNT1-MUT was inserted into the 3'UTR of the pMIR-reporter (Thermo Fisher Scientific, Waltham, USA) to package recombinant plasmids pMIR-WNT1-WT and pMIR-WNT1-MUT, respectively. Correctly identified recombinant plasmids WNT1-WT or WNT1-MUT were co-transfected into HEK293T cells with miR-140-5p or NC plasmids, respectively. After incubating for $48 \mathrm{~h}$, cells were cleaved in $1 \times$ passive lysate, and luciferase activity was assessed using a luciferase test kit (Promega, Madison, USA) using a dual-luciferase reporter assay system (Promega). The related target effect was shown as relative luciferase activity (the ratio of firefly luciferase intensity to that of renilla). Renilla luciferase activity was used as the internal reference.

\section{qRT-PCR}

Total RNA was first extracted using cold Trizol (Invitrogen). After the RNA concentration was measured, $1 \mu \mathrm{g}$ of total RNA was transcribed reversely using a cDNA Reverse Transcription Kit (Takara Biomedical Technology, Beijing, China). Quantitative real-time polymerase chain reaction (qRT-PCR) was carried out in an ABI7500 quantitative PCR instrument (Thermo Fisher Scientific) using a Fluorescence Quantitative PCR kit (Takara Biomedical Technology) according to the following procedure: predenaturation for $10 \mathrm{~min}$ at $95^{\circ} \mathrm{C}$ and 40 PCR cycles (denaturation for $15 \mathrm{~s}$ at $95^{\circ} \mathrm{C}$, annealing for $30 \mathrm{~s}$ at $56^{\circ} \mathrm{C}$, extension for $35 \mathrm{~s}$ at $72^{\circ} \mathrm{C}$ ). U6 and GAPDH were the internal references for miRNA and mRNAs, respectively. The results were analyzed with the $2^{-\Delta \Delta \mathrm{Ct}}$ method. All related primers were provided by the Jima Pharmaceutical Technology Co., Ltd. (Shanghai, China) and the primer sequences are listed in Table 1. The RT-PCR was carried out in strict accordance with the instructions, and all consumables used in the experiment were soaked in diethyl pyrocarbonate (DEPC) (Rongyue Biotechnology Co., Ltd., Shanghai, China) to inactivate the enzymes, and were sterilized at a high temperature.

\section{Western blot}

The protein in the cells was extracted using RIPA lysate (R0010; Solarbio Science \& Technology Co. Ltd, Beijing, China) containing phenylmethylsulfonyl fluoride (PMSF), and then incubated on ice for $30 \mathrm{~min}$; the supernatant was 
Table 1. Related primer sequences of qRT-PCR

\begin{tabular}{|c|c|}
\hline Name & Sequence \\
\hline WNT1 & $\begin{array}{c}\text { F: 5'-CGCTCTTCCAGTTCTCAGACAC-3' } \\
\text { R: 5'-CAGGATGGCAAAGGGTTCG-3' }\end{array}$ \\
\hline miR-140-5p & $\begin{array}{l}\text { F: 5'-GAGTGT-CAGTGGTTACCGT-3' } \\
\text { R: 5'-GCATGGTCC-GAGGTATTC-3' }\end{array}$ \\
\hline$\beta$-catenin & $\begin{array}{l}\text { F: 5'-GCCACAGGATTACAAGAAGC-3' } \\
\text { R: 5'-CCACCAGAGTGAAAAGAACG-3' } \\
\text { R: 5'-ATCCAGACTCTGACCTTTT-3' }\end{array}$ \\
\hline MMP-7 & $\begin{array}{l}\text { F: 5'-GGGATTAACTTCCTGTATGC-3' } \\
\text { R: 5'-GATCTCCATTTCCATAGGTTG-3' }\end{array}$ \\
\hline GAPDH & $\begin{array}{l}\text { F: 5'-GGGTGTGAACCATGAGAAGTATG-3' } \\
\text { R: 5'-GATGGCATGGACTGTGGTCAT-3' }\end{array}$ \\
\hline U6 & $\begin{array}{c}\text { F: 5'-CTCGGACTCAGCCTGCA-3' } \\
\text { R: 5'- TCAATATTACCGAGCTGCGT-3' }\end{array}$ \\
\hline
\end{tabular}

obtained by centrifuging at $12,000 \times \mathrm{g}$ for $10 \mathrm{~min}$ at $4^{\circ} \mathrm{C}$. The protein concentration was examined using a Pierce BCA Kit (23225; Thermo Fisher Scientific), and adjusted to equilibrium using deionized water. Forty-microgram protein samples were added to wells on SDS-PAGE gel and the proteins were separated at $80 \mathrm{~V}$ using 10\% SDS-PAGE gel (P0012A; Biyun Tian Biotechnology Research Institute, Shanghai, China) for $2 \mathrm{~h}$. The wet transfer method was used to transfer the protein to the polyvinylidene difluoride (PVDF) membranes (iseq00010; Merck Millipore, Burlington, USA), then the membranes were blocked for $2 \mathrm{~h}$ with tris-buffered saline with Tween 20 (TBST) buffer containing 5\% skimmed milk powder. After being washed 3 times using TBST buffer, the membranes were incubated with primary antibodies as listed, respectively: $\beta$-catenin rabbit antibody $(1: 1,000$, ab32572; Abcam, Cambridge, UK), WNT1 mouse antibody (1:500, ab15251; Abcam), C-myc rabbit antibody (1:1,000, 5605; Cell Signaling Technology, Leiden, the Netherlands), MMP-7 antibody (1:1,000, 71031; CST), and GAPDH rabbit monoclonal antibody (1:10,000, ab181602; Abcam) at $4^{\circ} \mathrm{C}$ overnight. After being washed 3 times using TBST buffer, the membranes were conjugated with diluted sheep antirabbit second antibody (1:2,000-1:50,000, ab205718; Abcam) and sheep anti-mouse second antibody (1:10,000, Jackson; Jackson Immunoresearch, USA) at room temperature for $90 \mathrm{~min}$. An electrochemiluminescence (ECL) fluorescence detection kit (Amersham, Little Chalfont, UK) was used. The membranes were treated with enhanced chemiluminescence reagent (Amersham) and imaged with the Bio-Rad image analysis system (Bio-Rad Laboratories Inc., Hercules, USA) in gel imager. The protein content was analyzed using Quantity One v. 4.6.2 software (Bio-Rad Laboratories Inc.) and relative protein content was shown as a grey value of a targeted protein band/gray value of a GAPDH band.

\section{CCK8 assay}

Digested SKOV3 and OVCAR3 cells were seeded into 96well plates, and the absorbance of cells in the $\mathrm{NC}$ group and the miR-140-5p mimic group were each measured 3 times.
After $0 \mathrm{~h}, 24 \mathrm{~h}, 48 \mathrm{~h}$, and $72 \mathrm{~h}$ of culture, cell absorbance was measured using a commercial CCK8 Kit (Solarbio Science \& Technology Co. Ltd.).

\section{Flow cytometry}

Cell apoptosis was evaluated using an Annexin V-FITC/PI Double Staining Kit (CA1050; Solarbio Science \& Technology Co. Ltd.) as follows: SKOV3 and OVCAR3 cells were centrifuged, re-suspended and then mixed with $5 \mu \mathrm{L}$ of Annexin V-FITC. The cell suspension was reacted with the $5 \mu \mathrm{L}$ of Annexin V-FITC added before in the dark at room temperature for $15 \mathrm{~min}$. Then, $5 \mu \mathrm{L}$ of propidium iodide (PI) was added 5 min before detection. Subsequently, $5 \mu \mathrm{L}$ of propidium iodide (PI) was added and reacted on ice in the dark for $5 \mathrm{~min}$, and then cell apoptosis was detected using Cube6 flow cytometry (Sysmex Partec GmbH, Münster, Germany).

The cell cycles of the NC group and the miR-140-5p mimic group were detected using a cell cycle DNA detection kit (Solarbio Science \& Technology Co. Ltd.) as follows: The cells were centrifuged and re-suspended after washing, and then centrifuged again to remove the supernatant. After being fixed and washed, the cells were gently and evenly blown and passed through the cell strainer to prevent agglomeration. The cells were re-suspended, bathed in water at $37^{\circ} \mathrm{C}$ for $30 \mathrm{~min}$, mixed with $400 \mu \mathrm{L}$ of PI staining solution, incubated in the dark at $4{ }^{\circ} \mathrm{C}$ for $30 \mathrm{~min}$, and detected using flow cytometry.

\section{Transwell migration and invasion assay}

After being transfected for $48 \mathrm{~h}$, the cells were starved in serum-free medium for another $24 \mathrm{~h}$. After being digested and washed, the cells were re-suspended in RPMI1640 medium with $10 \mathrm{~g} / \mathrm{L}$ of bovine serum albumin (BSA) (Thermo Fisher Scientific).

For the invasion assay, a transwell chamber was put into a 24-well plate. The upper chamber surface of the bottom membrane in the transwell chamber was coated with matrigel diluent (40111es08; Shanghai Yisheng Biotechnology Co., Ltd., Shanghai, China). After air-drying at room temperature, the chamber was cleaned twice using serum-free RPMI1640 medium (Gibco, Waltham, USA). The density of the cell suspension was adjusted to $1 * 10^{5} / \mathrm{mL}$, and $200 \mu \mathrm{L}$ of cell suspension was added to the upper chamber. At the same time, $600 \mu \mathrm{L}$ of complete medium accompanied with $10 \%$ FBS was added to the lower chamber. After $24 \mathrm{~h}$, the cells adhering to the upper surface were cleaned with cotton swabs, and the invading cells on the bottom surface were fixed with 4\% paraformaldehyde (Leigen Biotechnology Co. Ltd., Beijing, China) for $15 \mathrm{~min}$. After staining for $15 \mathrm{~min}$ with $0.5 \%$ crystal violet (Solarbio Technology Co. Ltd.), the cells were washed 3 times with PBS and 6 fields of vision were randomly selected to be photographed with an inverted microscope, and the cell number was counted. 
The migration assay was the same as the invasion assay, except that matrigel coating was not used on the transwell chambers.

\section{Immunofluorescence test}

The cells were inoculated into 24-well plates in which sterile cell climbing slices had already been placed. After $24 \mathrm{~h}$, the supernatant of the culture medium was discarded and the cells were fixed with $4 \%$ paraformaldehyde for $20 \mathrm{~min}$ and penetrated for $10 \mathrm{~min}$ by $0.1 \%$ Triton- 100 . The cells were then washed 3 times and blocked with $5 \%$ BSA before being incubated with $0.1 \mathrm{~mL}$ of $\beta$-catenin primary antibody (1:250, ab32572; Abcam) for $1 \mathrm{~h}$ at room temperature. After being washed 3 times, the cells were incubated with $0.1 \mathrm{~mL}$ of a fluorescent second antibody (ab150075; Abcam) in the dark for 30 min. After staining the nuclei with DAPI for $5 \mathrm{~min}$, the slides were carefully taken out after being washed 3 more times. Finally, the slides were sealed and observed under a microscope.

\section{Statistical analysis}

The statistical analysis was performed using SPSS v. 21.0 statistical software (SPSS Inc., Chicago, USA). Related measurement data was shown as means \pm standard deviation (SD). A t-test was used to compare differences between 2 groups and a one-way analysis of variance (ANOVA) with the Bonferroni correction was used to compare differences among multiple groups. A p-value of $<0.05$ indicated statistically significant differences.

\section{Results}

\section{Downregulation of miR-140-5p in human ovarian cancer cells}

With the aim of studying the effects of miR-140-5p on human ovarian cancer cells, the expression difference of miR-140-5p in the human epithelial ovarian cancer cell line SKOV3, the ovarian cancer cell line OVCAR3 and the human normal ovarian epithelial cell line IOSE80 was first assessed using qRT-PCR (Fig. 1). The results showed that the expression of miR-140-5p in both SKOV3 and OVCAR3 cells was sharply decreased compared with the expression in IOSE80 cells $(\mathrm{p}<0.05)$.

\section{Effects of miR-140-5p overexpression on the proliferation and apoptosis of human ovarian cancer cells}

To verify the impact of miR-140-5p on human ovarian cancer cells, the expression level of miR-140-5p in SKOV3 and OVCAR3 cells was first checked with qRT-PCR, and then cell proliferation was detected using a CCK8 assay

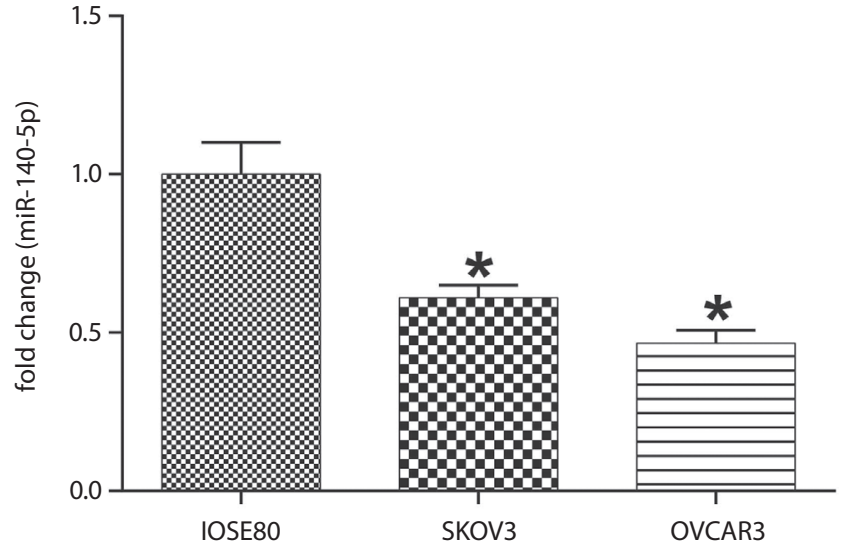

Fig. 1. miR-140-5p expression in human ovarian cancer cells and normal human ovarian epithelial cells compared with the IOSE80 group, ${ }^{*} p<0.05$

(Fig. 2A,B). The results showed that expression of miR$140-5 p$ was significantly upregulated in the miR-140-5p mimic group compared with the NC group $(\mathrm{p}<0.05)$. Correspondingly, the proliferation of miR-140-5p in the mimic group was significantly decreased compared with the NC group $(\mathrm{p}<0.05)$.

The cell cycle was further examined using flow cytometry (Fig. 2C). The cell cycle of the miR-140-5p mimic group was mainly blocked in the G0/G1 phase compared with the NC group ( $\mathrm{p}<0.05$ ). Cell apoptosis detection was conducted using annexin V-FITC/PI staining (Fig. 2D) and the proportion of early apoptotic cells was found to be significantly increased in the miR-140-5p mimic group compared with the NC group $(\mathrm{p}<0.05)$. These results made it clear that upregulated miR-140-5p suppressed proliferation and accelerated apoptosis in SKOV3 and OVCAR3 cells.

\section{Effects of miR-140-5p on migration and invasion of ovarian cancer cells}

Effects of miR-140-5p on the migration and invasion capacity of SKOV3 and OVCAR3 cells were evaluated using transwell assays (Fig. 3). The results showed that the migration and invasion capacity of SKOV3 and OVCAR3 cells were both largely inhibited by the emiR-140-5p mimic compared with the mimic NC $(\mathrm{p}<0.05)$.

\section{MiR-140-5p mediated the Wnt signal pathway by targeting WNT1}

Binding sites between miR-140-5p and WNT1 were first forecast using a bioinformatics analysis website (Fig. 4A) and then verified with a dual-luciferase reporter assay (Fig. 4B). Luciferase activity was strongly decreased in the co-transfection group of the miR-140-5p mimic and WNT1-WT compared with the NC group ( $\mathrm{p}<0.05)$, but luciferase activity was not obviously affected by the cotransfection of the miR-140-5p mimic and WNT1-MUT compared with that in the NC group. 
A

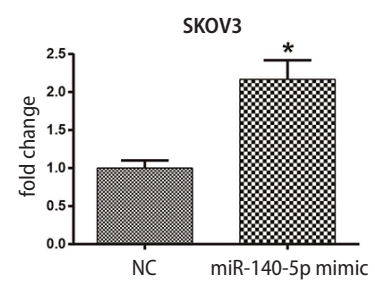

C

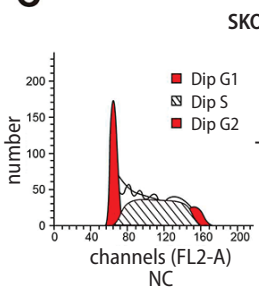

SKOV3

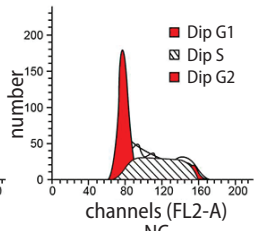

OVCAR3

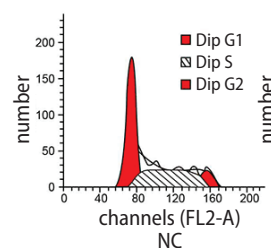

NC

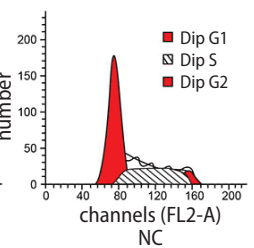

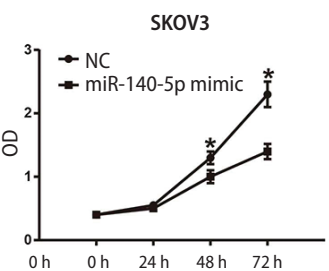

B

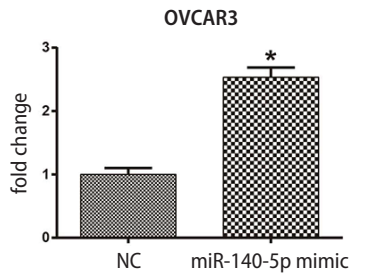

OVCAR3

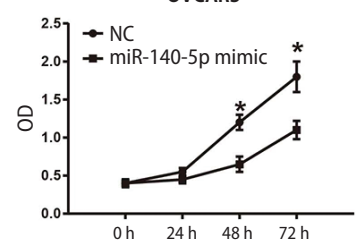

D

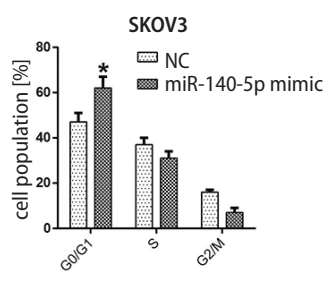

OVCAR3
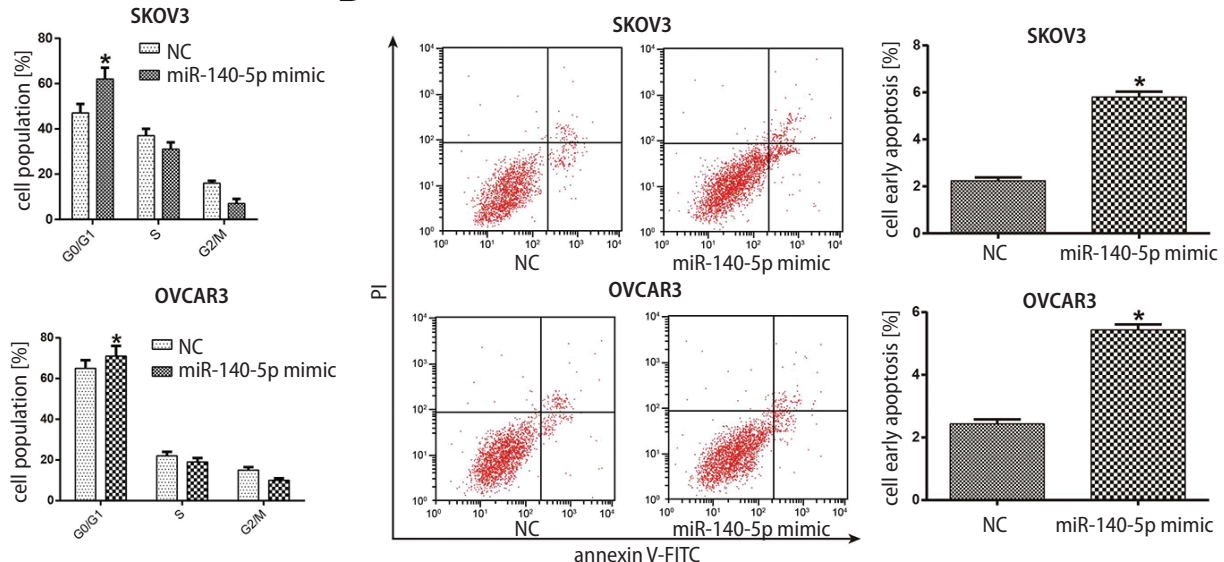

Fig. 2. Proliferation and apoptosis of ovarian cancer cells

A. miR-140-5p expression in SKOV3 cells and cell proliferation detection. B. miR-140-5p expression in OVCAR3 cells and cell proliferation detection. C. Cell cycle detected with flow cytometry and the corresponding column analysis. D. Cell apoptosis detected with flow cytometry and the corresponding column analysis. Compared with the NC group, ${ }^{*} \mathrm{p}<0.05$.

To further reveal the association between miR-140-5p and the Wnt signaling pathway, expressions of the Wnt signaling pathway-related $\beta$-catenin, WNT1 and associated downstream factors $C-m y c$ and $M M P-7$, which are closely related to tumor development, were evaluated using qRT-PCR (Fig. 4C) and western blot (Fig. 4F,G). The results showed that the expressions of $\beta$-catenin, WNT1, $C$-myc, and $M M P-7$ were all decreased in the miR-140-5p mimic group compared to the NC group $(\mathrm{p}<0.05)$.

The results of immunofluorescence in Fig. 4D and 4E showed that the number of cells with $\beta$-catenin transfer to the nucleus was significantly lower in the miR-140-5p mimic group than in the NC group, indicating that miR140-5p overexpression effectively suppresses nuclear transfer of $\beta$-catenin $(\mathrm{p}<0.05)$.

\section{Discussion}

From the perspective of molecular biology, we studied the role and mechanisms of miR-140-5p in ovarian cancer cells. We found that an miR-140-5p mimic largely reduced the expression level of Wnt signaling pathway-related signal molecules, suppressing their activity and thus promoting cell apoptosis and inhibiting the invasion and migration capabilities of ovarian cancer cells.

Our study found that miR-140-5p was weakly expressed in ovarian cancer cell lines. Zheng et al. also found that miR-140-5p was weakly expressed in gastric cancer tissues and cells compared with normal tissues and cells. ${ }^{17}$
Similar situations have also been demonstrated in breast cancer and cervical cancer, ${ }^{18,19}$ and downregulated miR140-5p has always presaged a poor prognosis. Analogously, we demonstrated that the overexpression of miR-140-5p could inhibit the proliferation, metastasis and development of ovarian cancer cell lines. MiR-140-5p has also demonstrated an ability to block the development of multiple cancers, including the proliferation and metastasis of gastric cancer cells. ${ }^{17}$ Besides, miR-140-5p has been shown to inhibit tumor metastasis and development by downregulating the expression of ADAM-related genes in hypopharyngeal and colorectal cancer. ${ }^{20,21}$ Moreover, miR-140-5p can not only inhibit the proliferation of synovial fibroblasts and inflammatory cytokine secretion by targeting TLR4, but can also inhibit proliferation and migration of glioma cells by targeting the VEGFA/MMP2 pathway. ${ }^{22,23}$ In conclusion, miR-140-5p can regulate the malignant behavior of various kinds of cancers, including ovarian cancer, and prevent further deterioration of ovarian cancer.

For our in-depth study of corresponding mechanisms of miR-140-5p in ovarian cancer, the potential signaling pathways regulated by miR-140-5p were predicted using a bioinformatics website, and it was found that there are binding sites between miR-140-5p and WNT1. The expressions of Wnt pathway-related $\beta$-catenin, WNT1, $C-m y c$, and $M M P-7$ were found to be significantly reduced in the miR-140-5p mimic group compared to the NC group, and the number of cells with $\beta$-catenin transfer to the nucleus in the miR-140-5p mimic group was also decreased significantly compared with the NC group. 
A

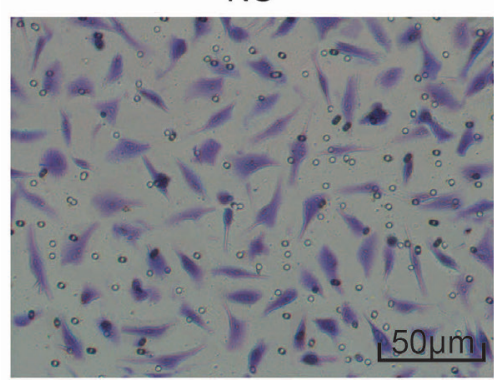

SKOV3

B
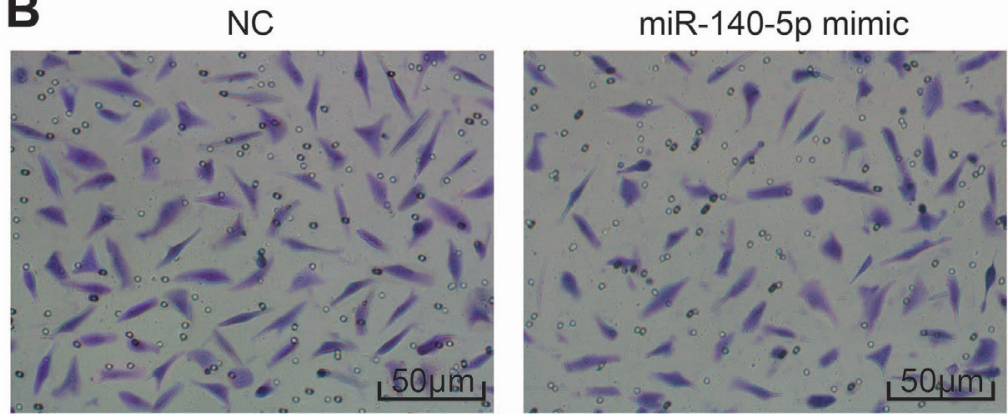

OVCAR3

C

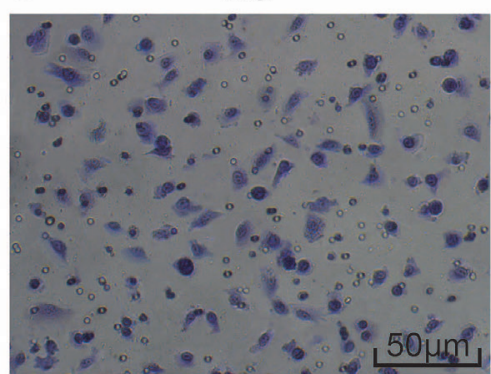

SKOV3

D
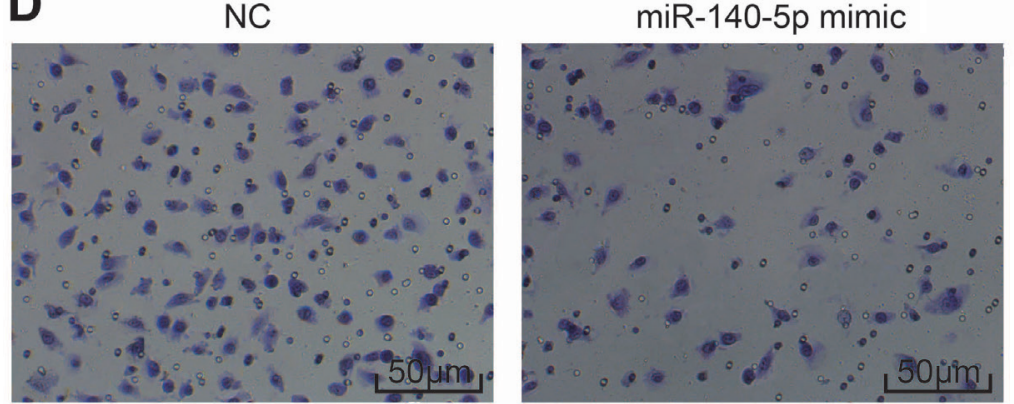

OVCAR3

miR-140-5p mimic

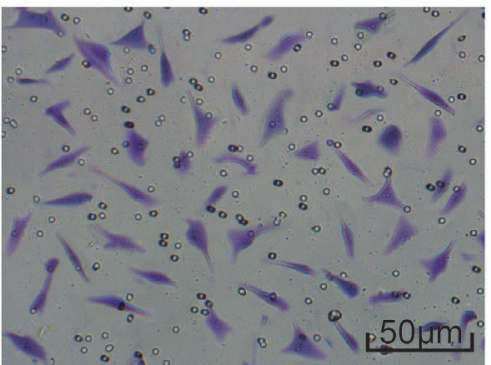

\section{skov}



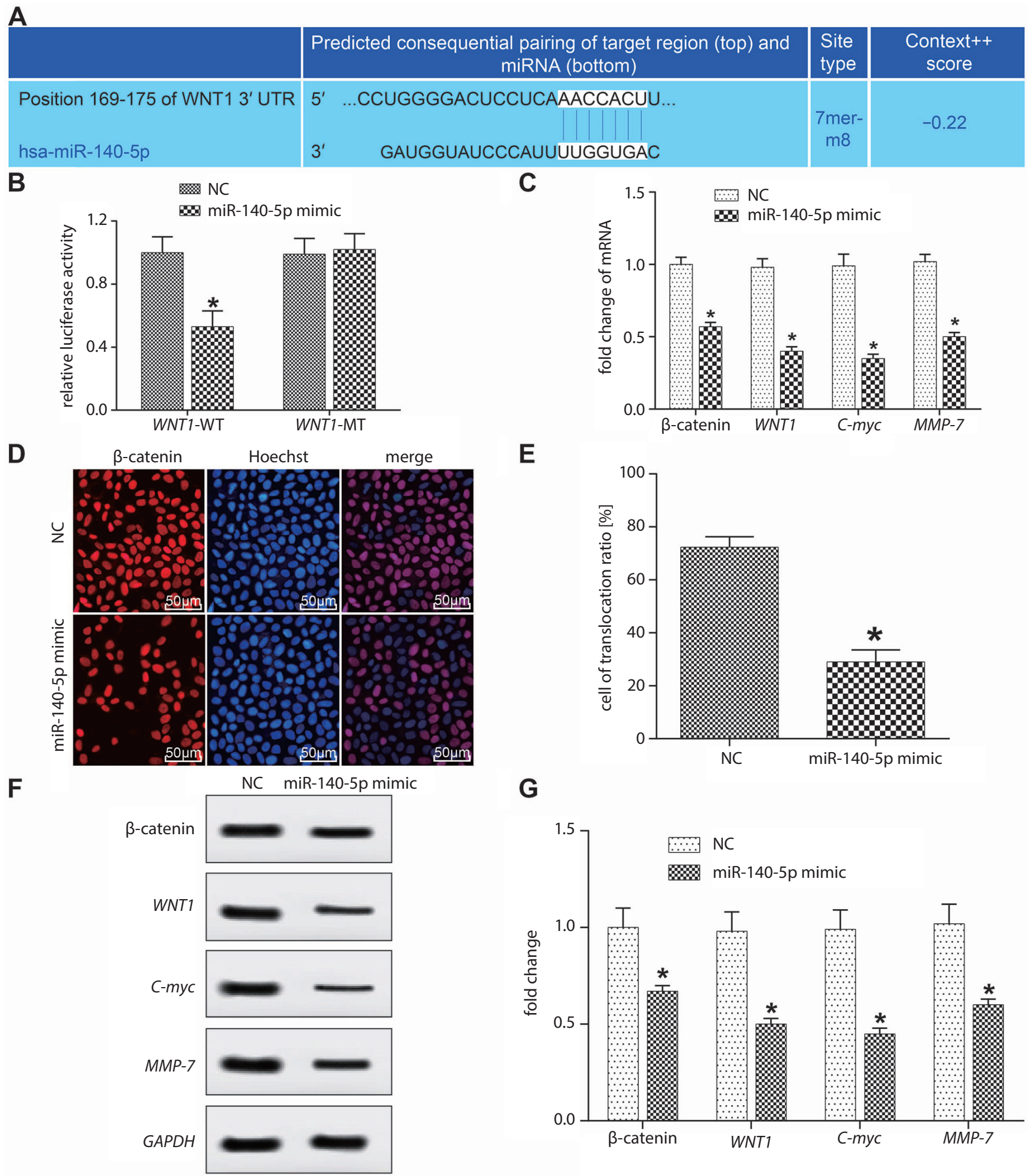

Fig. 4. miR-140-5p mediates the Wnt signaling pathway

A. Binding sites between miR-140-5p and WNT1 were analyzed using a bioinformatics analysis website. B. The relationship between miR-140-5p and WNT1 was further verified with a dual-luciferase reporter assay. C. The expression of Wnt signaling pathway-related signal molecules was detected using qRT-PCR. D and E. Nuclear transfer of $\beta$-catenin was detected using an immunofluorescence test. The corresponding histogram analysis is also shown. $\mathrm{F}$ and G. Expression of Wnt signaling pathway-related signal molecule genes was detected with western blot; the corresponding histogram analysis is also shown. Compared with the NC group, ${ }^{*} p<0.05$ 
WNT1 is an important regulatory gene of the Wnt signaling pathway, and overexpression of WNT1 has been shown to upregulate the expression of $\beta$-catenin, $C-m y c, M M P-7$, and related other factors, thus accelerating the proliferation and development of lung cancer cells. ${ }^{24}$ In most cases, the Wnt pathway activates its target genes by promoting $\beta$-catenin transfer into the nucleus. ${ }^{25}$ However, abnormal activation of the Wnt signal has the ability to regulate cancer stem cells and plays a key role in the production and deterioration of multiple types of cancer. ${ }^{26}$ Similarly, Wu et al. found that miR-140-5p can inhibit the Wnt signaling pathway so as to reduce cell proliferation and strengthen drug efficacy in breast cancer cells. ${ }^{27}$

Although we have shown that miR-140-5p regulates the proliferation, apoptosis, migration, and invasion ability of ovarian cancer cells by regulating WNT1, it is still unclear whether miR-140-5p could also regulate the progression of ovarian cancer through other signal factors. Thus, more experiments are needed to further explore the function and molecular mechanisms of miR-140-5p in ovarian cancer, in order to provide a more powerful basis for clinical application.

\section{Conclusions}

We found that miR-140-5p inactivates the Wnt/ $\beta$-catenin signaling pathway by regulating WNT1, and prevents further deterioration of ovarian cancer. MiR-140-5p is expected to provide novel ideas for the treatment of ovarian cancer.

\section{ORCID iDs}

Yunyu Wu (i) https://orcid.org/0000-0002-2975-5365

Jie Li (ㄷ) https://orcid.org/0000-0002-4734-1940

Shuying Chen (1) https://orcid.org/0000-0002-0148-0048

Zhiwu Yu (i) https://orcid.org/0000-0002-1870-1409

\section{References}

1. Webb PM, Jordan SJ. Epidemiology of epithelial ovarian cancer. Best Pract Res Clin Obstet Gynaecol. 2017;41:3-14.

2. Heintz APM, Odicino F, Maisonneuve P, et al. Carcinoma of the ovary. FIGO $26^{\text {th }}$ Annual Report on the Results of Treatment in Gynecological Cancer. Int J Gynaecol Obstet. 2006;95(Suppl 1):S161-S192.

3. Reid BM, Permuth JB, Sellers TA. Epidemiology of ovarian cancer: A review. Cancer Biol Med. 2017;14(1):9-32.

4. LiW, Liu Z, Chen L, Zhou L, Yao Y. MicroRNA-23b is an independent prognostic marker and suppresses ovarian cancer progression by targeting runt-related transcription factor-2. FEBS Lett. 2014;588(9):1608-1615.

5. Chen W, Huang L, Hao C, et al. MicroRNA-155 promotes apoptosis in SKOV3, A2780, and primary cultured ovarian cancer cells. Tumour Biol. 2016;37(7):9289-9299.
6. Li X, Chen W, Zeng W, et al. microRNA-137 promotes apoptosis in ovarian cancer cells via the regulation of XIAP. Br J Cancer. 2017;116(1):66-76.

7. Wang M, He Y, Shi L, Shi C. Multivariate analysis by Cox proportional hazard model on prognosis of patient with epithelial ovarian cancer. Eur J Gynaecol Oncol. 2011;32(2):171-177.

8. Li Y, Chen Y, Li J, et al. Co-delivery of microRNA-21 antisense oligonucleotides and gemcitabine using nanomedicine for pancreatic cancer therapy. Cancer Sci. 2017;108(7):1493-1503.

9. Li P, Sun Y, Liu Q. MicroRNA-340 induces apoptosis and inhibits metastasis of ovarian cancer cells by inactivation of NF-x03BA;B1. Cell Physiol Biochem. 2016;38(5):1915-1927.

10. Kan $Q$, Su Y, Yang H. MicroRNA-335 is downregulated in papillary thyroid cancer and suppresses cancer cell growth, migration and invasion by directly targeting ZEB2. Oncol Lett. 2017;14(6):7622-7628.

11. Liu C, Liu R, Zhang D, et al. MicroRNA-141 suppresses prostate cancer stem cells and metastasis by targeting a cohort of pro-metastasis genes. Nat Commun. 2017;8:14270-14270.

12. Santos JC, Lima NDS, Sarian LO, et al. Exosome-mediated breast cancer chemoresistance via miR-155 transfer. Sci Rep. 2018;8(1):829-829.

13. Flamini V, Jiang W, Cui Y. Therapeutic role of MiR-140-5p for the treatment of non-small cell lung cancer. Anticancer Res. 2017;37(8):4319-4327.

14. Zhai H, Fesler A, Ba Y, Wu S, Ju J. Inhibition of colorectal cancer stem cell survival and invasive potential by hsa-miR-140-5p mediated suppression of Smad2 and autophagy. Oncotarget. 2015;6(23):19735-19746.

15. Yang $\mathrm{P}$, Xiong J, Zuo L, Liu K, Zhang H. miR-140-5p regulates cell migration and invasion of non-small cell lung cancer cells through targeting VEGFA. Mol Med Rep. 2018;18(3):2866-2872.

16. Dihlmann S, von Knebel Doeberitz M. Wnt/beta-catenin-pathway as a molecular target for future anti-cancer therapeutics. Int J Cancer. 2005:113(4):515-524.

17. Fang Z, Yin S, Sun R, et al. miR-140-5p suppresses the proliferation, migration and invasion of gastric cancer by regulating YES1. MolCancer. 2017;16(1):139-139.

18. Lu Y, Qin T, Li J, et al. MicroRNA-140-5p inhibits invasion and angiogenesis through targeting VEGF-A in breast cancer. Cancer Gene Ther. 2017;24(9):386-392.

19. Su Y, Xiong J, Hu J, et al. MicroRNA-140-5p targets insulin like growth factor 2 mRNA binding protein 1 (IGF2BP1) to suppress cervical cancer growth and metastasis. Oncotarget. 2016;7(42):68397-68411.

20. Jing $P, S a N, X u W$. miR-140-5p affects the migration and invasion of hypopharyngeal carcinoma cells by downregulating ADAM10 expression [in Chinese]. Zhonghua Er Bi Yan Hou Tou Jing Wai Ke Za Zhi. 2016;51(3):189-196.

21. Yu L, Lu Y, Han X, et al. microRNA-140-5p inhibits colorectal cancer invasion and metastasis by targeting ADAMTS5 and IGFBP5. Stem Cell Res Ther. 2016;7(1):180.

22. Li H, Guan S, Lu Y, Wang F. MiR-140-5p inhibits synovial fibroblasts proliferation and inflammatory cytokines secretion through targeting TLR4. Biomed Pharmacother. 2017;96:208-214.

23. Hu Y, Li Y, Wu C, et al. MicroRNA-140-5p inhibits cell proliferation and invasion by regulating VEGFA/MMP2 signaling in glioma. Tumour Biol. 2017;39(4):1010428317697558. doi:10.1177/1010428317697558.

24. Huang CL, Liu D, Ishikawa S, et al. Wnt1 overexpression promotes tumour progression in non-small cell lung cancer. Eur J Cancer. 2008; 44(17):2680-2688.

25. Lustig B, Behrens J. The Wnt signaling pathway and its role in tumor development. J Cancer Res Clin Oncol. 2003;129(4):199-221.

26. Duchartre Y, Kim YM, Kahn M. The Wnt signaling pathway in cancer. Crit Rev Oncol Hematol. 2016;99:141-149.

27. Wu D, Zhang J, Lu Y, et al. miR-140-5p inhibits the proliferation and enhances the efficacy of doxorubicin to breast cancer stem cells by targeting Wnt1. Cancer Gene Ther. 2019;26(3-4):74-82. 